\title{
Utilização de gentamicina no tratamento de neonatos atendidos em uma maternidade pública da região metropolitana de Belo Horizonte, Minas Gerais (1999)
}

\author{
Maria Auxiliadora Parreiras Martins" ${ }^{* 1}$, Sérgia Maria Starling1, Siura Aparecida Borges ${ }^{2}$ \\ ${ }^{1}$ Faculdade de Farmácia, Universidade Federal de Minas Gerais, ${ }^{2}$ Universidade de Itaúna
}

${ }^{*}$ Correspondência:

M. A. P. Martins

R. Nascimento Gurgel, ํㅜ750 apto 501

B. Grajaú

30430-340 - Belo Horizonte - MG

E-mail: auxiliadoramartins@ig.com.br
A gentamicina é um antimicrobiano de baixo custo amplamente utilizado no tratamento da septicemia neonatal. Entretanto, existe um risco potencial de oto e nefrotoxicidade. Neste trabalho, buscouse caracterizar o recém-nascido hospitalizado em uso de gentamicina e avaliar o cumprimento do protocolo de tratamento adotado em uma Instituição pública de Belo Horizonte, Minas Gerais. Trata-se de um estudo transversal incluindo 63 recémnascidos atendidos em uma maternidade pública no periodo de agosto, outubro e dezembro de 1999. Essa maternidade é referência na assistência à gestação e ao parto de alto risco em Minais Gerais. A maioria dos recém-nascidos foi prematura $(85,7 \%)$ e de baixo peso $(90,5 \%)$. Apenas dois (3,2\%) casos de septicemia foram confirmados por hemocultura. O protocolo adotado preconiza a administração de doses de 2,5 $\mathrm{mg} / \mathrm{kg}$ a intervalos de 24 horas para neonatos com peso inferior a $1200 \mathrm{~g}$ e 12 horas para aqueles com peso superior a esse. Observou-se que o esquema terapêtico foi adotado em 96,7\% dos casos. Contudo, constatou-se uma utilização por tempo superior ao recomendado, frente à hemocultura negativa, em mais de 70,0\% dos casos.
Unitermos

- Gentamicina

- Esquema terapêutico

- Septicemia neonatal

- Protocolo de tratamento

\section{INTRODUÇÃO}

Os aminoglicosídeos são antimicrobianos usados regularmente no tratamento da septicemia neonatal precoce (observada até 72 horas de vida) e tardia (observada após 72 horas de vida), associados principalmente a antimicrobianos $\beta$-lactâmicos. $\mathrm{O}$ uso destes fármacos é direcionado para tratar infecções causadas por micorganismos gram-negativos, importantes agentes etiológicos da septicemia (Bertino et al., 1994).
O baixo potencial de indução à resistência bacteriana e seu custo reduzido em relação aos fármacos mais novos elegem esta classe terapêutica como primeira escolha para o tratamento da septicemia neonatal (Bertino et al., 1994). Por outro lado, o baixo índice terapêutico desta classe e reações adversas relacionadas à nefro e ototoxicidade são fatores limitantes de sua utilização (Tavares, 1999). Para minimizar estes riscos que podem ser potencializados devido à imaturidade das funções hepática e renal ao nascimento, grandes esforços são 
mobilizados com o objetivo de se estabelecer condições seguras para o uso destes fármacos.

Na prática clínica, a dosagem sérica constitui um parâmetro útil para avaliar a eficácia e minimizar o risco de toxicidade (Bertino et al., 1994). Contudo, esta prática nem sempre pode ser adotada devido às dificuldades operacionais, pois exige infra-estrutura e recursos humanos especializados. Assim, a adoção de protocolos de tratamento que assegurem a eficácia terapêutica e reduzam o potencial tóxico são de grande utilidade para otimizar a farmacoterapia do paciente.

Os protocolos podem ser definidos como formas estruturadas de suporte do manejo técnico que incluem a definição de objetivos terapêuticos e seqüência temporal de cuidados e estratégias diagnósticas e terapêuticas (Polanczyk et al., 2003). O estabelecimento de protocolos de tratamento permite padronizar as diretrizes clínicas na abordagem das doenças, reduzir os erros na aplicação das escollhas terapêuticas (Atallah, Castro, 1998) e melhorar a prática clínica (Grimshaw, Russel, 1993). Na assistência perinatal, Osório-de-Castro et al. (2002) observaram que a elaboração de protocolos de uso de antiinfecciosos em unidade de terapia intensiva neonatal permitiu uma padronização da conduta terapêutica racionalizando o uso destes medicamentos.

Na atualidade, a adoção de protocolos é considerada uma medida essencial para subsidiar a decisão terapêutica baseada na eficácia e segurança (Malick et al., 1998; Cremesp, 2000) à luz das evidências científicas (Wilson et al., 1995; Naylor, Guyatt, 1996; Atallah, Castro, 1998; Smith, Duncan, 1999) com o menor custo possível (Eccles, Mason, 2001). Entretanto, o sucesso de um protocolo depende de uma série de fatores que vão desde os métodos utilizados para sua elaboração e divulgação até a adesão propriamente dita do corpo clínico (Thomson et al., 1995; Wilson et al., 1995). Assim, o cumprimento dos protocolos torna-se um importante indicador de processo, na avaliação da qualidade em serviços de saúde, permitindo nortear medidas de intervenção e contribuir na melhoria da atenção ao paciente (Thomson et al., 1995; Costa et al., 1998).

O presente estudo teve como objetivo caracterizar o recém-nascido hospitalizado em uso de gentamicina em uma maternidade pública de Belo Horizonte e avaliar a adesão ao protocolo em vigor para a utilização da gentamicina na Instituição.

\section{MATERIAL E MÉTODOS}

Este estudo foi realizado em maternidade pública da região metropolitana de Belo Horizonte, que exerce, junto ao Sistema Único de Saúde - SUS, o papel de referên- cia na assistência à gestação e ao parto de alto risco no estado de Minas Gerais. O presente estudo consiste em análise retrospectiva, que utilizou o prontuário médico como fonte para coleta de dados. Utilizou-se como critério de inclusão todos os casos de septicemia neonatal ocorridos na maternidade nos meses de agosto, outubro e dezembro de 1999. Foram excluídos os pacientes cujo prontuário não foi localizado ou se apresentava incompleto com falta da maioria dos dados relacionados ao paciente e às características de utilização do medicamento. Nos casos em que os dados de caracterização do neonato estavam presentes, porém os relativos à utilização de gentamicina encontravam-se incompletos, apenas as informações disponíveis foram incluídas na análise.

Os critérios diagnósticos para septicemia neonatal adotados pelo corpo clínico da Instituição são sinais clínicos e / ou laboratoriais de infecção com ou sem confirmação por hemoculturas. Dentre os sinais e sintomas sugestivos de septicemia neonatal os mais significativos são: a dificuldade respiratória, apnéia, hipoatividade, hipotonia, hiper ou hipoglicemia, febre ou hipotermia, vômitos, distensão abdominal, petéquias e baixa perfusão tecidual. Também são avaliados os fatores de risco para a infecção, destacando-se: baixo peso ao nascer, ruptura prolongada de membranas (superior a 24 horas), febre materna, líquido amniótico purulento, prematuridade, asfixia perinatal e infecção urinária materna.

O Serviço de Controle de Infecção Hospitalar $\mathrm{SCIH}$ forneceu a lista de pacientes neonatais que utilizaram gentamicina no período estudado. A busca dos prontuários destes pacientes foi feita junto ao Serviço de Arquivo Médico - SAME, que os disponibilizou para análise. As variáveis pesquisadas nos prontuários estão representadas nas Tabelas I e II.

TABELA I - Variáveis pesquisadas relativas às características dos recém-nascidos em uso de gentamicina na maternidade pública de Belo Horizonte, 1999

\begin{tabular}{ll}
\hline Características dos recém-nascidos \\
\hline I & número de registro \\
II & data de nascimento \\
III & sexo \\
IV & gemelaridade \\
V & peso ao nascer \\
VI & $\begin{array}{l}\text { idade gestacional e método utilizado para sua } \\
\text { determinação }\end{array}$ \\
VII & classificação relacionando peso e idade gestacional \\
VIII & APGAR (um e cinco minutos) \\
IX & comprimento e perímetro cefálico \\
X & tipo de parto \\
XI & hipótese diagnóstica \\
\hline
\end{tabular}


TABELA II - Variáveis pesquisadas relativas às características ao tratamento com gentamicina dos recémnascidos atendidos na maternidade pública de Belo Horizonte, 1999

\begin{tabular}{ll}
\hline \multicolumn{2}{l}{ Variáveis } \\
\hline I & idade ao iniciar o tratamento \\
II & peso ao iniciar o tratamento \\
III & dose \\
IV & intervalo posológico \\
V & tempo de uso \\
VI & peso ao final do tratamento \\
VII & ocorrência de ajuste de dose (baseado na alteração \\
& de peso) \\
VIII & presença de diurese \\
IX & confirmação da infecção/método confirmatório \\
X & uso concomitante de outros fármacos. \\
\hline
\end{tabular}

Foi realizado um pré-teste para avaliar os formulários para coleta de dados. Nesta etapa, incluíram-se 10 prontuários selecionados por técnica de amostragem aleatória simples, cuja coleta permitiu adequar os formulários de acordo a necessidade de rearranjo ou inclusão de campos de preenchimento. Os pacientes que participaram do estudo piloto foram posteriormente incluídos no estudo principal.

A hipótese diagnóstica registrada foi aquela aventada ao nascer. Outras hipóteses diagnósticas observadas e confirmadas durante a utilização de gentamicina também foram registradas.

Os pacientes que apresentaram agravamento do quadro durante o período de internação foram transferidos da maternidade para o Centro de Terapia Intensiva - CTI de outras instituições e retornaram à maternidade de origem após a melhora do quadro. A coleta de dados referente a estes pacientes foi realizada de acordo com o prontuário médico e o relatório de transferência. Embora em alguns casos não houvesse maior detalhamento da utilização de gentamicina, os dados disponíveis foram coletados e incluídos na pesquisa.

O protocolo para utilização de gentamicina na Instituição preconiza a administração de doses de $2,5 \mathrm{mg} / \mathrm{kg}$ de $24 \mathrm{em} 24$ horas para pacientes com peso inferior a $1200 \mathrm{~g}$ e de 12 em 12 horas para pacientes com peso superior a esse. O protocolo recomenda a suspensão da antibioticoterapia no caso de hemocultura inicial negativa (após três dias de tratamento) com evolução clínica favorável.

A avaliação da adesão ao protocolo foi realizada comparando-se o tratamento adotado, relatado no prontuário médico, com o protocolo da Instituição. Verificou-se se havia um seguimento das diretrizes recomendadas considerando dose, intervalo posológico e tempo de tratamento.

A análise de dados foi feita utilizando-se o programa Epi-Info, versão 6.0 (Dean et al., 1995). Foi realizada uma análise estatística descritiva envolvendo dados numéricos absolutos e relativos associados às variáveis anteriormente descritas.

\section{RESULTADOS}

Os nascidos vivos atendidos pela maternidade totalizaram 239 no mês de agosto, 289 no mês de outubro e 241 no mês de dezembro de 1999. Os recém-nascidos com suspeita de infecção nestes meses foram $27(11,3 \%)$, $26(9,0 \%)$ e $15(6,2 \%)$, respectivamente. A taxa média de casos de infecção em relação aos nascidos vivos foi de $8,8 \%$.

Do total de 68 casos, 63 prontuários foram localizados e incluídos no estudo. Destes, 50 continham todos os dados investigados. Dos 13 restantes, 2 se apresentavam incompletos e 11 pacientes foram transferidos e não puderam ser acompanhados integralmente pelo prontuário médico. A hemocultura foi realizada nos 63 (100\%) casos envolvendo suspeita de infecção sendo que a maioria $(96,8 \%)$ foi tratada por septicemia clínica sem confirmação microbiológica.

A maioria dos pacientes estudados iniciou a utilização de gentamicina nas primeiras 72 horas de vida, totalizando $62(98,4 \%)$ recém-nascidos, dos quais 54 $(87,1 \%)$ iniciaram antes de completar 24 horas de vida.

Dos neonatos estudados, $39(61,9 \%)$ eram do sexo feminino e $24(38,1 \%)$ eram do sexo masculino. Foram observados $7(11,1 \%)$ casos de gemelaridade. De acordo com o tipo de parto, $31(49,20 \%)$ pacientes nasceram de parto vaginal e os outros $32(50,8 \%)$ de parto cesariana. Os recém-nascidos prematuros totalizaram $54(85,7 \%)$ pacientes. Não foram observados nascimentos pós-termo. A distribuição dos neonatos segundo o peso associado com a idade gestacional pode ser observada na Figura 1.

A maioria dos pacientes estudados apresentou baixo peso ao nascer totalizando 57 (90,5\%) pacientes (Figura 2).

A determinação da idade gestacional foi obtida segundo a data da última menstruação em 52 casos $(82,5 \%)$ sendo este o método de maior precisão para esta determinação. Nos outros casos, foi obtida pelo método de Capurro em 4, pela ultrasonografia tardia em 4 e pelo método de New Ballard em 3 casos. Em relação às hipóteses diagnósticas, observou-se total de $61(96,8 \%)$ pacientes com distúrbios respiratórios, $54(85,7 \%)$ com icterícia, $7(11,1 \%)$ com distúrbios metabólicos, 2 (3,2\%) com 
alterações cardíacas, $1(1,6 \%)$ com alterações neurológicas e outros quadros diversos foram apresentados por 22 (34,9\%) pacientes. O número total de hipóteses diagnósticas foi superior ao número de neonatos estudados, pois em alguns casos os pacientes apresentavam mais de uma hipótese diagnóstica.

De acordo com a Figura 3, observa-se que houve adesão ao protocolo em 59 (96,7\%) dos prontuários analisados no que se refere à dose e ao intervalo posológico. Dois casos foram retirados da análise pois os dados refe-
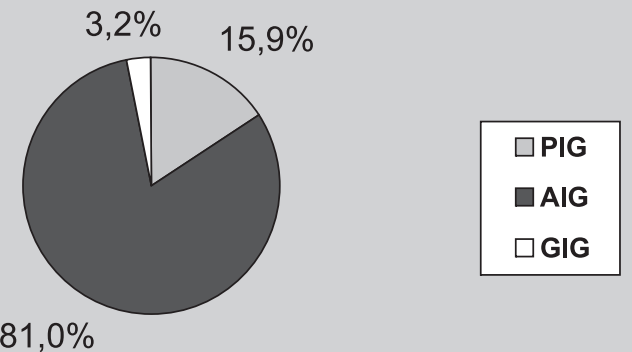

\section{Pequeno para a idade gestacional - PIG Adequado para a idade gestacional - AIG Grande para a idade gestacional - GIG}

FIGURA 1 - Distribuição dos neonatos segundo o peso associado com a idade gestacional na Maternidade pública, Belo Horizonte, 1999 rentes ao regime posológico não estavam disponíveis no prontuário médico.

Não foi possível obter informações sobre a realização de ajuste de dose para 11 pacientes devido à sua transferência para outra instituição durante parte do tratamento. Dos 52 pacientes restantes, observou-se que o ajuste de dose de acordo com a alteração diária de peso foi efetuado em $46(88,5 \%)$ casos. A diurese estava presente em $59(93,7 \%)$ pacientes sendo que no restante da amostra esta informação não foi registrada.

Quanto à duração do tratamento, observou-se que metade dos neonatos estudados foi tratada por um período de até 6 dias (Tabela III), sendo um paciente retirado da análise devido à falta de informação no prontuário médico. Apenas $2(3,2 \%)$ casos apresentaram hemocultura positiva justificando um tratamento por tempo superior a 7 dias. No entanto, metade da amostra foi tratada por mais de 7 dias, sendo a suspensão do antibiótico em apenas 15 casos $(24,2 \%)$ devido à hemocultura negativa.

Com relação à utilização concomitante de outros fármacos com a gentamicina, observou-se a associação $\operatorname{com} \beta$-lactâmicos em 100,0\% dos casos. A associação com cefalosporinas, vancomicina e indometacina foi observada neste estudo com freqüência inferior a $4 \%$. Outra associação observada foi aquela com atropina e pancurônio com freqüência de $1,6 \%$ e $4,8 \%$, respectivamente.

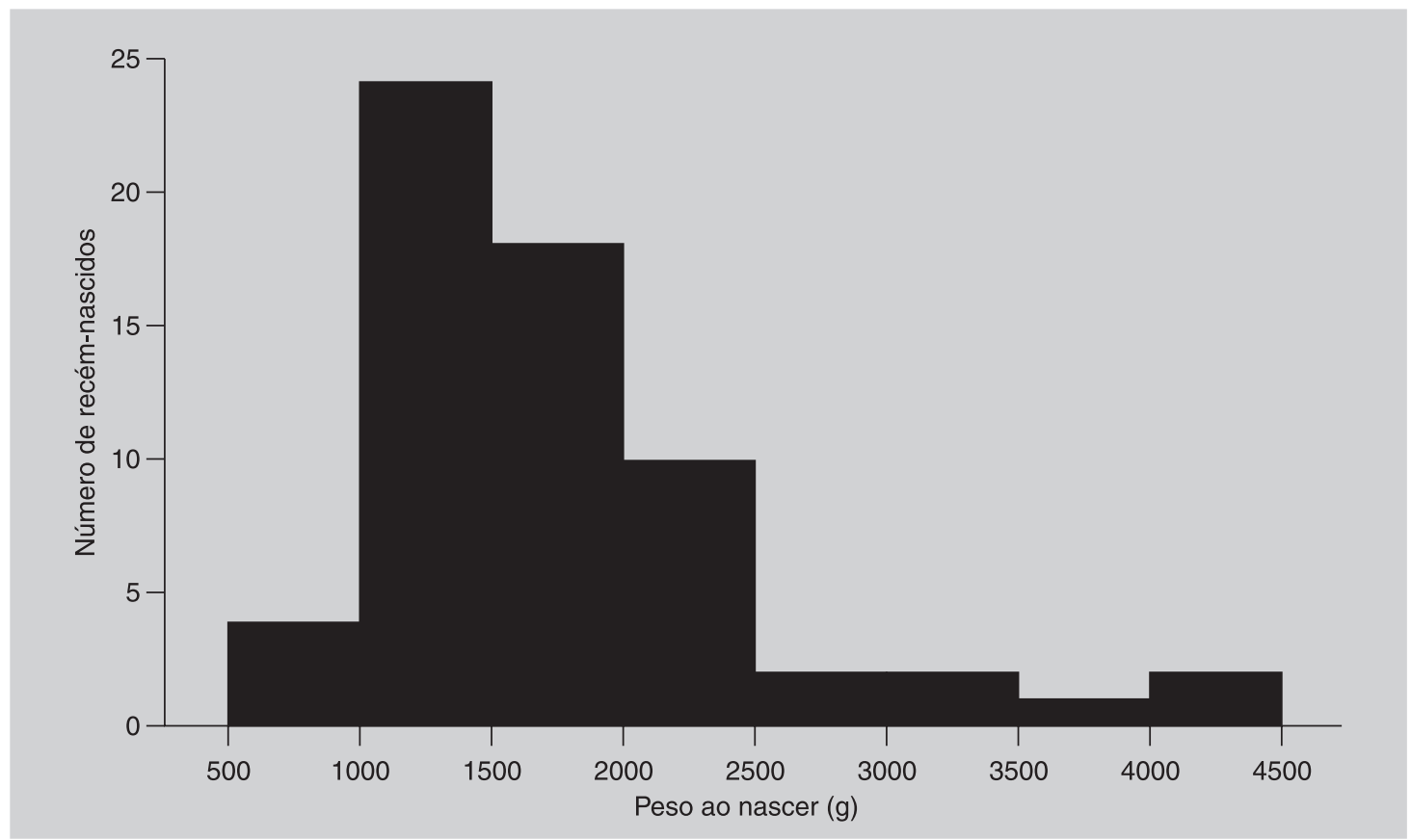

FIGURA 2 - Distribuição de peso dos recém-nascidos tratados com gentamicina na maternidade pública, Belo Horizonte, 1999 


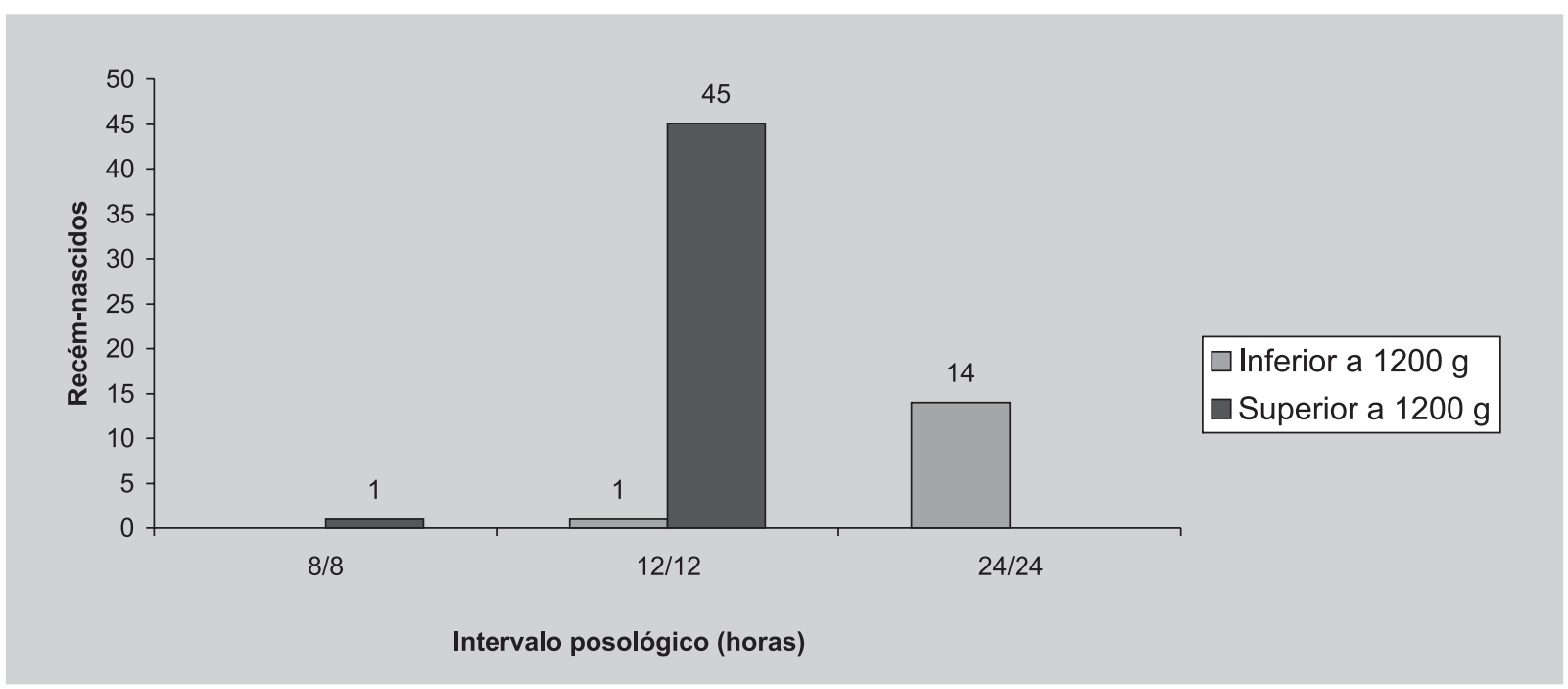

FIGURA 3 - Intervalos posológicos para utilização de gentamicina de acordo com a faixa de peso dos recém-nascidos atendidos na maternidade pública de Belo Horizonte, 1999

TABELA III - Duração do tratamento com gentamicina dos neonatos estudados, Belo Horizonte, 1999

\begin{tabular}{ccc}
\hline Duração do tratamento(dias completos) & n & Freqüência acumulada (\%) \\
\hline Até 3 dias & 15 & 24,2 \\
De 4 a 6 dias & 16 & 50,0 \\
De 7 a 14 dias & 30 & 98,4 \\
Superior a 14 dias & 01 & 100,0 \\
Total & 62 & \\
\hline
\end{tabular}

\section{DISCUSSÃO}

A maternidade onde foi realizado o estudo é uma Instituição pública considerada referência na assistência à gestação e ao parto de alto risco oferecendo cuidados especializados aos recém-nascidos provenientes desses quadros. A Instituição considera aceitável a faixa de um a oito por cento de incidência de septicemia neonatal, sendo que a incidência observada no período estudado $(8,8 \%)$ foi superior ao limite máximo. A variação mensal da incidência de septicemia neonatal apresentada em relação ao número de nascidos vivos não foi investigada.

Segundo o serviço de neonatologia da Instituição, o índice de cesarianas idealmente realizado por maternidades de alto risco deve estar na faixa de 30,0\%. A taxa geral de cesarianas realizadas na maternidade em estudo durante 1999 foi de 32,5\%. É possível que a elevada taxa observada nesta amostra $(50,8 \%)$ se deva ao agrupamento de mulheres em gestação de maior complexidade nas quais foi necessário este tipo de intervenção.

Os recém-nascidos em uso de gentamicina amostrados nesta maternidade pública apresentaram como características básicas a prematuridade e o baixo peso ao nascer. Estas características têm sido apontadas pela literatura como fatores de risco associados à septicemia neonatal (Gerdes, Polin, 1998). Contudo, a maioria destes neonatos apresentaram peso adequado para a idade gestacional. A elevada freqüência de complicações respiratórias apresentada pelos pacientes era esperada estando relacionada à prematuridade.

A septicemia neonatal é um quadro grave no qual a implementação de antibioticoterapia precoce, observada na maioria dos casos, justifica-se pelo seu alto índice de mortalidade, podendo ser fatal qualquer atraso no início do tratamento (Gerdes, Polin, 1998). A gentamicina associada a um antibiótico $\beta$-lactâmico é, em geral, o tratamento de primeira eleição para septicemia neonatal (Bertino et al., 1994). Portanto, a seleção do antimicrobiano e a decisão pela antibioticoterapia precoce são procedimentos adequadamente adotados nesta maternidade. O esquema posológico adotado, no que se refere à dose e intervalo posológico, foi seguido em $96,7 \%$ dos casos. Nos dois 
casos em que esse protocolo não foi seguido houve a redução do intervalo posológico o que está associado a um maior risco de toxicidade (Mattie et al., 1989; Barclay et al., 1994). Razões específicas para esta conduta não foram investigados.

Contudo, observou-se uma baixa adesão ao protocolo no que diz respeito ao tempo de tratamento. O protocolo adotado preconiza no máximo três dias de antibioticoterapia no caso de recém-nascidos sem confirmação microbiológica do diagnóstico de septicemia e com evolução clínica favorável. Observou-se baixo índice de positividade das hemoculturas incluindo apenas dois $(3,2 \%)$ casos, porém $72,6 \%$ dos neonatos com hemocultura negativa foram tratados por mais de três dias, sendo que para $50 \%$ o tratamento superou os 7 dias e um paciente foi tratado durante 16 dias. Existem divergências na literatura quanto à duração do tratamento da septicemia neonatal sendo recomendado um período variável de sete a 14 dias, se houver confirmação por hemoculturas (Saíz-liorens, Mccracken, 1995; Yurdakok, 1998). Não foram feitos levantamentos mais detalhados das indicações para prolongamento do tratamento em nenhum dos casos. Entretanto, a manutenção da antibioticoterapia nos casos de hemocultura negativa parece sugerir um baixo grau de confiança no exame laboratorial, ou uma baixa correlação desse com a evolução clínica dos pacientes. Em qualquer dos casos, a prática deve ser objeto de uma avaliação rigorosa, pois o prolongamento da antibioticoterapia pode estar associada ao aumento do risco de reações adversas no recém-nascido, seleção de cepas resistentes e aumento dos custos de tratamento.

O recém-nascido, em geral, perde peso após o nascimento antes de iniciar o processo de ganho de peso ponderal. Assim, é de grande importância ajustar a dose após a redução de peso para evitar o uso de doses maiores do que as necessárias, com aumento do risco de toxicidade do fármaco. Nesse sentido, observou-se que em $88,5 \%$ dos casos este procedimento foi adotado, o que reflete a atenção do corpo clínico em estabelecer segurança no cálculo da dose do antibiótico. A presença de diurese em $93,7 \%$ dos casos também deve ser considerada, pois está associada à função renal ativa do neonato, contribuindo para a eliminação do fármaco.

$\mathrm{O}$ alto índice de associação da gentamicina com antimicrobianos $\beta$-lactâmicos era esperado já que esta leva ao aumento do efeito bactericida e do espectro de ação antibacteriano. Os riscos de nefrotoxicidade, por efeito aditivo, podem ser elevados pela associação da gentamicina com medicamentos nefrotóxicos como é o caso de cefalosporinas, vancomicina e indometacina. Outras associações potencialmente perigosas observadas neste estudo são aquelas com agentes bloqueadores neuromusculares não despolarizantes, como atropina e pancurônio (Tavares, 1999). Assim, respeitando-se as indicações, estes casos merecem cuidadosa monitorização para reduzir o risco de complicações.

A dose média de gentamicina utilizada foi de $2,5 \mathrm{mg} / \mathrm{kg}$ a qual representa a dose convencionalmente empregada (Saíz-liorens, McCracken, 1995). O ajuste de dose é realizado segundo o peso que é apontado como o melhor parâmetro correlacionado ao clearance total e ao volume total de distribuição da gentamicina em neonatos (Murphy et al., 1998). Embora a idade gestacional também possa ser um importante determinante da maturidade renal (Langlass, Mickle, 1999) este parâmetro pode não ser ideal para estimar o clearance total, pois muitas pacientes não informam com precisão a data da última menstruação, o que impossibilita uma aferição precisa da idade gestacional.

Para o estabelecimento de esquemas posológicos adequados, é importante compreender as características farmacocinéticas dos aminoglicosídeos as quais estão relacionadas com as suas propriedades terapêuticas (Zhanel, Craig, 1994). Este grupo de fármacos apresenta atividade bactericida dependente da concentração plasmática (Moore et al., 1987; Mattie et al., 1989; Kumana, Yuen, 1994; Freeman et al., 1997) tendo como principais determinantes da eficácia: a dose total, a área sob a curva da concentrção plasmática - ASC e a relação entre os picos plasmáticos e a concentração inibitória mínima CIM (Moore et al., 1987; Mattie et al., 1989; Skopnik et al., 1991).

A literatura tem sugerido que uma dose única ao dia entre 3,5 e $5 \mathrm{mg} / \mathrm{kg}$ parece ser igualmente efetiva, quando comparada à dose convencional de $2,5 \mathrm{mg} / \mathrm{kg}$ em intervalos menores (Murphy et al., 1998; Romero et al., 1998; Langlass, Mickle, 1999; Lundergan et al., 1999; Thureen et al., 1999). A adoção de doses maiores em administração única diária poderia assegurar picos plasmáticos mais elevados que garantem uma maior ASC, maior relação entre os picos plasmáticos e a CIM do microrganismo infectante (Moore et al., 1987; Mattie et al., 1989; Skopnik et al., 1991) e um efeito pós-antibiótico mais prolongado (Barclay et al., 1994) necessários à manutenção da eficácia. Além destes fatores, a administração única diária preservaria o recém-nascido de maior freqüência de manipulação.

A maternidade adota um protocolo que preconiza duas administrações diárias e tem obtido um alto índice de adesão $(96,7 \%)$ por parte do corpo clínico no que se refere à dose e intervalo posológico. Esta conduta é reforçada pela falta de estudos conclusivos em neonatologia so- 
bre a efetividade e segurança de esquemas terapêuticos com doses maiores administradas uma vez ao dia. Contudo, constatou-se baixa adesão ao protocolo no que se refere ao prolongamento do tratamento em caso hemocultura negativa $(72,6 \%)$.

Vários trabalhos têm sido conduzidos para avaliar a adesão e o impacto da implementação dos protocolos de tratamento (Grimshaw, Russel, 1993; Araújo, 2000; Tobin et al., 2001;). Tobin et al. (2001) e Polanczyk et al. (2003) observaram que transcorrido um determinado período de tempo após a implantação do protocolo houve um retrocesso dos indicadores de seguimento do mesmo. É necessário, então, aliar constantemente estratégias de educação e avaliação para contribuir na adesão aos protocolos (Curry, 2000; Smith, 2000; Ribeiro, 2002).

\section{CONCLUSÃO}

Este estudo permitiu verificar que a maioria dos neonatos com diagnóstico de septicemia atendidos na maternidade pública no período estudado é prematura $(85,7 \%)$ e de baixo peso $(90,5 \%)$, porém com peso adequado para a idade gestacional $(80,9 \%)$. Observou-se alta taxa de cesarianas $(50,8 \%)$ e uma taxa de suspeita de sepse superior ao esperado pela Instituição $(8,8, \%)$. Embora tenha sido observado alto índice de adesão ao protocolo de tratamento no que se refere à dose e ao intervalo posológico, detectou-se prolongamento da antibioticoterapia nos casos de hemocultura negativa. Este resultado é de grande importância para a Instituição no sentido de fundamentar futuras discussões acerca do protocolo adotado e da condução do tratamento da septicemia neonatal.

\section{ABSTRACT \\ Gentamicin utilization for treating neonates at a public maternity in Belo Horizonte, Minas Gerais (1999)}

Gentamicin is a low cost antimicrobian commonly used in the treatment of neonatal sepsis. Nevertheless, there is a potential risk of oto and nephrotoxicity. The objectives of the study were to characterize the hospitalized neonate in use of gentamicin and to evaluate the follow-up of the dosing protocol adopted. It is a cross sectional study of a total of 63 neonates who received assistence at a public maternity in August, October and December, 1999. It is a Brazilian public institution which plays a role of reference in the high risk pregnancy and delivery. Most patients were premature (85.7\%) and low birth weight (90.5\%). Only two (3.2\%) sepse cases were confirmed by hemoculture. The protocol adopted recommends doses of $2.5 \mathrm{mg} / \mathrm{kg}$ with a 24 hour dosing interval for neonates weighing less than $1200 \mathrm{~g}$ and a 12 hour dosing interval for those with a superior weight. Almost all doctors (96.7\%) followed the protocol considering the dose and dosing interval. However, most patients (72.6\%) with negative hemoculture were treated for more than the recommended time.

UNITERMS: Gentamicin. Dosing regimen. Neonatal sepsis. Treatment protocol

\section{REFERÊNCIAS BIBLIOGRÁFICAS}

ARAÚJO, R.Q. Antibiótico-profilaxia em cirurgias ortopédicas: resultado da implantação de um protocolo. Campinas, 2000. 70p. [Dissertação de Mestrado. Faculdade de Ciências Médicas. Universidade Estadual de Campinas].

ATALLAH, A.N.; CASTRO, A.A. Medicina baseada em evidências: o elo entre a boa ciência e a boa prática clínica. Diagn. Trat., São Paulo, v.3, n.2, p. 50-58, 1998.

BARCLAY, M.L.; BEGG, E.J.; HICKLING, K.G. What is the evidence for once-daily aminoglycoside therapy? Clin. Pharmacokinet., Auckland, v.27, n.1, p.32-48, 1994.

BERTINO, J.S.; RODVOLD, K.A.; DESTACHE, C.J. Cost considerations in therapeutic drug monitoring of aminoglycosides. Clin. Pharmacokinet., Auckland, v.26, n.1,p.71-81, 1994.

COSTA, J. S.D.; ROWAN V.R.; LUZ, R.M.; MANZOLI P.P.; CORRÊA, P.; RECUPERO, L.F. Auditoria médica: avaliação de alguns procedimentos inseridos PAISM no posto de saúde da vila municipal, Pelotas, RS, Brasil. Cad. Saúde Públ., Rio de Janeiro. v.14, n.1, p. 43-49, 1998.

\section{CONSELHO REGIONAL DE MEDICINA DO ESTADO} DE SÃO PAULO - Cremesp. Avaliação dos serviços de assistência ao parto e ao neonato no estado de São Paulo, 1997-1998. São Paulo: CREMESP, 2000.91p.

CURRY, S. J. Organizational interventions to encourage guideline implementation. Chest, Northbrook, v.118, suppl.2, p.40S-46S, 2000. 
DEAN, A.G.; DEAN, J.A.; BURTON, A.H.; DICKER, R.C. Epi-info, version 6: a word processing, data base, and statistics program for public health on Ibm-compatible microcomputers. Atlanta: Centers for Disease Control and Prevention, 1995.

ECCLES, M.; MASON, J. How to develop cost-conscious guidelines. Health Technol., Assess., v.5, p.1-69, 2001.

FREEMAN, C.D.; NICOLAU, D.P.; BELLIVEAU, P.P.; NIGHTINGALE, C.H. Once-daily dosing of aminoglycosides: review and recommendations for clinical practice. J. Antimicrob. Chemother., London, v.39, p.677-686, 1997.

GERDES, J.S.; POLIN, R. Early diagnosis and treatment of neonatal sepsis. Indian J. Pediatr., v.65, n.1, p.63-78, 1998.

GRIMSHAW, J.M.; RUSSEL, I. Effect of clinical guidelines on medical practice: a systematic review of rigorous evaluations. Lancet, London, v.342, p.1317-1322, 1993.

KUMANA, C.R.; YUEN, K.Y. Parenteral aminoglycoside therapy: selection, administration and monitoring. Drugs, v.47, n.6, p.902-913, 1994.

LANGLASS, T.M.; MICKLE, T.R. Standard gentamicin regimen in neonates. Am. J. Health-Syst. Pharm., v.56, n.5, p.440-443, 1999.

LUNDERGAN, S.F.; GLASSCOCK, G.F.; KIM, E.H. Once-daily gentamicin dosing in newborn infants. Pediatrics, Elk Grove Village, v.103, n.6, p.1228-1234, 1999.

MALICK, A.M. Indicadores. In: QUALIDADE na gestão local de serviços e ações de saúde. São Paulo: FSP-USP, 1998. cap.5, p.52-58.

MATTIE, H.; CRAIG, W.A.; PECHÈRE, J.C. Determinantes of efficacy and toxicity of aminoglycosides. $J$. Antimicrob. Chemother., London, v.24, p.281-293, 1989.

MOORE, R.D.; LIETMAN, P.S.; SMITH, C.R. Clinical response to aminoglycoside therapy: importance of the ratio of peak concentration to minimal inhibitory concentration. J. Infect. Dis., Chicago, v.155 p.93-99, 1987.
MURPHY, J.E.; AUSTIN, M.L.; FRYE, R.F. Evaluation of gentamicin pharmacokinetics and dosing protocols in 195 neonates. Am. J. Health-Syst. Pharm., Bethesda, v.55, n.21, p.2280-2288, 1998 .

NAYLOR, C.D.; GUYATT, G.H. User's guides to the medical literature: XI. How to use an article about a clinical utilization review. JAMA., v. 275, n. 18, p. 14351439, 1996.

OSÓRIO-DE-CASTRO, C.G.S.; PEIXOTO, M.A.P.; CASTILHO, S.R. Câmbio na assistência perinatal como determinante de consumo e diversidade de antiinfecciosos? Estudo de UTI neonatal no Rio de Janeiro. Cad. Saúde Públ., v.18, n.1, p.257-267, 2002.

POLANCZYK, C.A.; KUCKENBECKER, R.; CAYE, L.; VACARO, R.; FERREIRA, J.; PROMPT, C.A. Informatização de protocolos assistenciais como estratégia de adesão às melhores práticas clínicas. Disponível em $<$ http://www.avesta.com.br/anais/dados/ trabalhos/323.pdf>. Acesso em: 07 set. 2003.

RIBEIRO, E. Avaliação do uso de um instrumento educativo para solicitação de albumina humana em um hospital universitário de São Paulo. São Paulo, 2002. 185p. [Tese de Doutorado - Faculdade de Ciências Farmacêuticas. Univerisidade de São Paulo].

ROMERO, C.A.; CASTILLO, E.G.; SECADES, C.M.; LÓPEZ, L.A.; VALIENTE, P.S. Once daily gentamicin dosing in neonates. Pediatrics Infect. Dis. J., Baltimore, v.17, n.12, p.1169-1171, 1998.

SAÍZ-LIORENS X.; MCCRACKEN, G.H. Clinical pharmacology of antibacterial agents. In: REMINGTON, J.S.; KLEIN, J.O. (Eds.). Infectious disease of the fetus and newborn infant. 3.ed. Philadelphia: WB Saunders Company, p. 325-338, 1995.

SKOPNIK, H.; WALLRAF, R.; NIES, B. et al. Pharmacokinetics and antibacterial activity of daily gentamicin. Arch. Dis. Child., London, v.67, p.57-61, 1991.

SMITH, W. Evidence for the effectiveness of techniques to change physician behavior. Chest, Northbrook, v.118, suppl.2,p.8S-17S, 2000. 
SMITH, M.I.; DUNCAN, B.B. Epidemiologia clínica e a medicina baseada em evidências. In: ROUQUAYROL, M.Z.; ALMEIDA FILHO, N. Epidemiologia e Saúde. 5.ed. Rio de Janeiro: Medsi, 1999. p.183-206.

TAVARES, W. Manual de antiobióticos e quimioterápicos antiinfecciosos. 2.ed. Belo Horizonte: Atheneu, 1999. $792 \mathrm{p}$.

THOMSON, R.; LAVENDER, M.; MADHOK, R. How to ensure that guidelines are effective. $B M J$, London, v.311, p.237-242, 1995.

THUREEN, P.J.; REITER, P.D.; GRESORES, A.; STOLPMAN, N.M.; KAWATO, K.; HALL, D.M. Once- versus twice-daily gentamicin dosing in neonates ${ }^{3} 34$ weeks' gestation: cost-effectiveness analyses. Pediatrics, Elk Grove Village, v.103, n.3, p.594-598, 1999.
TOBIN, S.N.; CAMPBELL D.A.; BOYCE, N.W. Durability of response to a targeted intervention to modify clinician transfusion practice in a major teaching hospital. Med. J. Aust., Glebe, v.174, p.445-448, 2001.

WILSON, M.C.; HAYWARD, R.S.A.; TUNIS, S.R.; BASS, E.B.; GUYATT, G. Users' guide to the medical literature. VIII. How to use clinical practice guidelines. B. what are recommendations and will they help you in caring for your patients? JAMA, Chicago, v.274, n.20, p. 1630$1632,1995$.

YURDAKOK, M. Antibiotic use in neonatal sepsis. Turk. $J$. Pediatr., v.40, n.1, p.17-33, 1998.

ZHANEL, G.G.; CRAIG, W.A. Pharmacokinetic contributions to postantibiotic effects - focus on aminoglycosides. Clin. Pharmacokinet., Auckland, v.27, n.5, p.377-392, 1994.

Recebido para publicação em 16 de outubro de 2002. Aceito para publicação em 14 de março de 2005. 